\title{
Alterações histopatológicas associadas à braquiterapia intra-arterial com o samário-153
}

\author{
Histophatologics changes associated to intraarterial brachytherapy with samarium-153 \\ Dalton Bertolim Précoma'; Lúcia de Noronha²; Álvaro Vieira Moura ${ }^{3}$; Airton Seiji Yamada4; José Knopfholz5; \\ Dario Maciel Hauer6; Cesar Dusilek7; Rita Perussolo ${ }^{8}$; Márcia Olandoski'; José Claudio Meneghetti ${ }^{10}$
}

unitermos
Radiolesão vascular
Braquiterapia
Samário-153

Células xantomatosas

\section{resumo}

A lesão da parede arterial provocada por balão de angioplastia ou implante de próteses endovasculares em modelos experimentais e humanos pode provocar a reestenose do vaso, principalmente por migração e proliferação de células musculares lisas e síntese de matriz extracelular. Vários estudos demonstraram que a braquiterapia intra-arterial atua nestes fatores e, conseqüentemente, no tratamento da reestenose coronariana. Este estudo tem por objetivo avaliar as alterações vasculares morfológicas e morfométricas induzidas pela braquiterapia com samário-153 $\left({ }^{153} \mathrm{Sm}\right)$, utilizando uma dose considerada ideal e outra elevada em coelhos hipercolesterolêmicos. Foram analisados 43 coelhos hipercolesterolêmicos e um total de 86 artérias ilíacas submetidas à lesão por balão de angioplastia, divididos em três grupos, sendo irradiados com as doses de 15Gy $(n=14)$ e 60Gy $(n=36)$ e controle $(n=36)$. Foram realizadas análise morfométrica (área neo-intimal, área da camada média, área do vaso) e análise histológica qualitativa para avaliação tecidual. O colesterol médio foi de $1.362 \pm 497 \mathrm{mg} / \mathrm{dl}$ nos três grupos. O achado mais relevante foi uma significativa inibição da hiperplasia neo-intimal no grupo irradiado com a dose de 15Gy, maior do que o controle e com a dose de 60Gy. Na dose de $60 \mathrm{~Gy}$, além de ineficaz para inibir a proliferação neo-intimal, teve características tissulares e estruturais sugestivas de radiolesão, como presença de células xantomatosas, tecidos hialino e amorfo, proliferação vascular. Estas células, bem como o aumento das dimensões morfométricas do vaso, foram proporcionais aos graus de lesão nas lâminas elásticas interna e externa, sendo observados principalmente com a dose de 60Gy. Os segmentos médios das artérias ilíacas, representando o local de maior contato com o balão, tiveram maiores alterações morfométricas e celulares em relação aos segmentos referenciais (proximal e distal) nos três grupos. Em conclusão, o grupo de artérias submetidas à irradiação de 15Gy foi eficaz para a inibição da proliferação neo-intimal. O grupo irradiado com a maior dose de 60Gy foi ineficaz para inibir a hiperplasia neo-intimal e apresentou características morfométricas e teciduais compatíveis com a radiolesão vascular.

\section{abstract}

Balloon angioplasty or stents performed in animal models or in humans helps determine vessel restenosis. The brachytherapy inhibits the proliferation and migration of vascular smooth muscle cells and reduces neointimal formation, and works reducing extracellular matrix synthesis. Many studies showed that the endovascular brachytherapy is usefull for that factors. The purpose of this study is to evaluate the efficacy of Samarium-153 to inhibit intimal neoproliferation (IN) in hypercholestherolemic rabbits by using both an ideal doses and a higher one. Samarium-153 is a gamma and mainly beta emitting radioactive agent. Eighty-six iliac arteries of fortythree hypercholesterolemic rabbits underwent balloon angioplasty injury. They were divided in three different groups: control group $(C G=36)$, a group submitted to $15 \mathrm{~Gy}$ irradiation doses $(I C 15 \mathrm{~Gy}=14)$ and the third group irradiated with 60Gy (IG 60Gy =36). Quality histological and morphometric (neointimal, media layer and vessel areas) analysis were performed. Blood cholesterol levels $(1362 \pm 497 \mathrm{mg} / \mathrm{dl})$ were measured in the three groups. The most interesting finding was the significant inhibition of IN in the IG 15Gy comparing with CG and IG 60Gy. There was also reduction of the media layer and vessel areas in IG15Gy showing significant difference comparing to other groups. Medium and higher degrees xantomatous cells in the media layer were mostly found in IG 60Gy, as well as hyaline tissue and vascular proliferation. The presence of xantomatous cells as well as the increase of the morphometric size of the vessels were proportional to the lesion levels of the internal elastic layer. These changes were more frequently found in IG 60Gy. As a conclusion, we can state that the IG $15 \mathrm{~Gy}$ was effective to inhibit the IN while the IG 60Gy was not. The IG 60Gy presented histological and morphometric changes similar to those of vascular radio injury. key words

Vascular radio injury

Brachytherapy

Samarium-153

Xantomatous cells

1. Doutor em Cardiologia da Faculdade de Medicina da Universidade de São Paulo (FMUSP); professor-assistente de Cardiologia da Pontifícia Universidade Católica do Paraná (PUC.PR).

2. Doutora e professora de Patologia da PUC.PR.

3. Doutor e professor de Cardiologia da PUC.PR.

4. Médico nuclear do Centro de Radioimunoensaio e Medicina Nuclear do Paraná (Cermen).

5. Médico residente do Hospital Cajuru - PUC.PR.

6. Médico hemodinamicista.

7. Médico residente de Hemodinâmica do Hospital Nossa Senhora das Graças.

8. Física do Cermen.

9. Responsável pelo Setor de Bioestatística da pós-graduação da PUC.PR.

10. Chefe da disciplina de Medicina Nuclear do Instituto do Coração da FMUSP. 


\section{Introdução}

A reestenose é um grande desafio na hemodinâmica desde o início da angioplastia ${ }^{(1,2)}$. Constitui-se em tentativa de reparo tecidual frente à lesão da parede arterial, cuja resposta se caracteriza por neoproliferação intimal (NPI) e remodelamento da parede arterial. Neste mecanismo fisiopatogênico, destacam-se também a migração e a proliferação de células musculares lisas (CML) e o depósito de matriz extracelular, que, associados a fenômenos inflamatórios e proliferativos, formam a NP|(3-5).

A radiação ionizante usada em doenças neoplásicas também tem sido utilizada ultimamente na reestenose coronariana ${ }^{(6,7)}$. A irradiação provoca modificações fenotípicas que impedem a replicação celular pelo ácido desoxirribonucléico (DNA), inibindo a proliferação e a migração das CML e diminuindo o grau da hiperplasia neointimal por meio da emissão de radiação localizada ${ }^{(7)}$. Nos últimos sete anos, vários estudos experimentais e clínicos têm demonstrado a eficácia das radiações gama e beta na reestenose $\mathrm{e}^{(5,8)}$. Este estudo teve por objetivo analisar as alterações vasculares morfológicas e morfométricas induzidas pela braquiterapia com samário-153 $\left({ }^{153} \mathrm{Sm}\right) \mathrm{em}$ coelhos hipercolesterolêmicos.

\section{Método}

\section{Modelo animal}

Foram utilizados 43 coelhos brancos da raça New Zealand, com uma média de 4 meses de idade e peso de 2,6 $\pm 0,5 \mathrm{Kg}$. Foram alimentados com dieta hipercolesterolêmica a 2\% 14 dias antes do procedimento e mantida até o dia do sacrifício.

\section{Procedimento}

Os animais foram anestesiados e suas artérias femorais esquerda e direita expostas para introdução de um balão de angioplastia ( $20 \mathrm{~mm}$ de comprimento por $3 \mathrm{~mm}$ de diâmetro) até a artéria ilíaca. Neste local o balão foi insuflado com solução salina isotônica com pressão de 5 atm pelo período de 5 minutos em 18 coelhos (36 artérias) que constituíram o grupo controle (GC). O mesmo procedimento foi repetido em sete coelhos (14 artérias), sendo que nestes o balão foi insuflado com 15Gy de ${ }^{153} \mathrm{Sm}$ (GI 15Gy) por 13min e 38s. No terceiro grupo, com 18 coelhos ( 36 artérias), o balão foi insuflado com 60Gy (Gl 60Gy) por 6min e 22seg no primeiro dia e $8 \mathrm{~min}$ e 30 s no segundo dia. Nesta fase foi colhido sangue para a dosagem de colesterol.
Após $30 \pm 1$ dias, os animais foram sacrificados com dose letal de barbitúricos. Imediatamente após, foi realizada uma laparotomia com acesso a aorta e artérias ilíacas, as quais foram lavadas com formaldeído a $10 \%$ durante 10 minutos. Foi retirado o bloco composto de aorta terminal e artérias ilíacas, fixadas em formalina e encaminhadas para análise histológica (Figura 1).

\section{Preparo das amostras}

Após fixação por 24 horas, foram retiradas três a seis amostras de cada artéria ilíaca em cortes transversais, com 2 a $3 \mathrm{~mm}$ de espessura cada, representando assim os segmentos proximais, mediais e distais em ambas artérias, em todos os animais. Os segmentos mediais representavam a área central de maior contato com o balão de angioplastia. Os segmentos proximais e distais da artéria ilíaca de cada lado, denominados referenciais, correspondiam às extremidades em relação à área central (segmento medial). Também foram obtidas amostras aleatórias de segmentos de aorta. O material foi processado conforme técnica convencional, sendo obtidas 110 lâminas coradas com hematoxilina-eosina (HE) e elástica de Hardt (orceína) e 521 cortes transversais das artérias ilíacas (Figura 2).

\section{Análise histológica qualitativa}

Todas as lâminas foram analisadas de maneira cega, em microscópio de cinco cabeças Olympus ${ }^{\circledR} \mathrm{BX} 40$, sendo observadas as seguintes variáveis em todos os cortes realizados: a) graus de lesão vascular; b) graus de lesão da lâmina elástica interna (LEI) e lâmina elástica externa (LEE); c) alterações tissulares gerais: c1) proliferação vascular, (2) recanalização vascular, c3) material amorfo hialino, c4) teleangiectasia, c5) características do endotélio, c6) tecido de granulação e

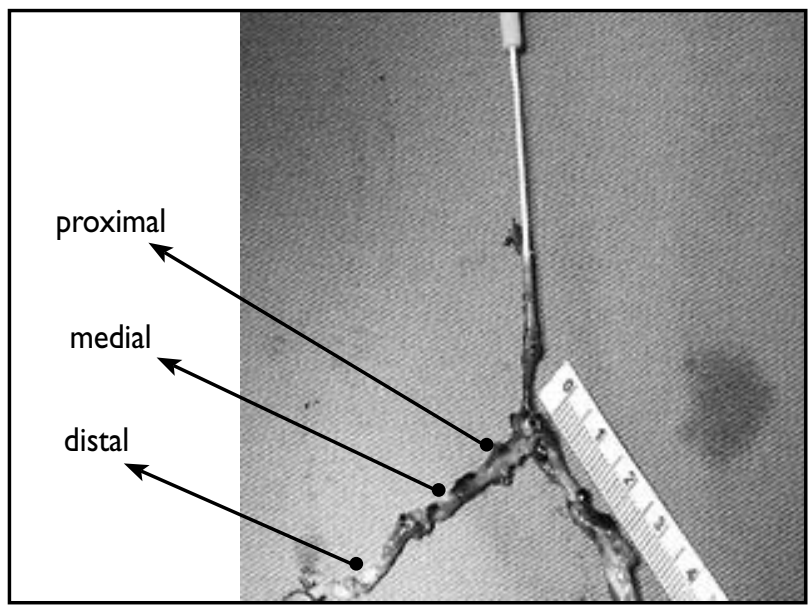

Figura 1 - Esquema demonstrando o bloco aorta/ilíacas e o resultado de seus cortes transversais 


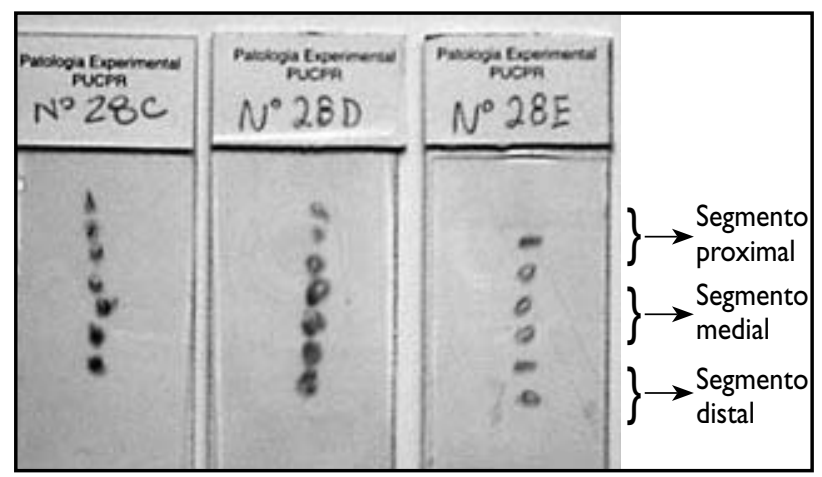

Figura 2 - Demonstrativo da disposição dos cortes nas lâminas dos três segmentos (proximal, medial e distal)

fibrose, c7) atipias celulares, c8) células xantomatosas, c9 ) trombose vascular e c10) necrose fibrinóide.

Estas variáveis foram classificadas conforme o escore exposto na Tabela 1.

\section{Análise histológica morfométrica (quantitativa)}

A leitura morfométrica foi realizada a fim de se determinar o lúmen arterial, a LEI, a LEE, a área da neo-íntima (ANI), a área da camada média (ACM) e a área do vaso. A leitura das lâminas foi realizada por meio do programa Pro Image-plus ${ }^{\circledR} \mathrm{v}$. 4,5 para Windows $s^{\circledast}$, acoplado a microscópio Olympus ${ }^{\circledR}$ BX50 e câmara de vídeo Sony ${ }^{\circledR}$, utilizando-se o aplicativo chamado morfometria de área, calibrado previamente em $\mu \mathrm{m}$ com a objetiva de quatro vezes. As medidas foram transferidas para o programa Excel Windows ${ }^{\circledast}$. As medidas morfométricas foram realizadas em todas as secções transversais da lâmina, no sentido proximal do corte, próximo da identificação da lâmina para o distal. Foram desprezados os segmentos com artefatos que impossibilitavam as medidas.

Para avaliar a área luminal (AL), a ANI, a ACM e a área do vaso, foram utilizados os seguintes critérios (Figura 3):

a) AL: determinada por uma linha traçada junto ao perímetro da borda luminal; b) ANI: determinada por uma linha traçada sob a borda luminal e pela linha do perímetro da LEI, isto é, AL-LEl;

c) ACM: determinada pelo perímetro da LEl e da LEE, isto é, LEE-LEl;

d) Área do vaso: compreendendo a área da LEE mais a ANI (LEE + ANI).

\section{Análise estatística}

A análise estatística foi realizada utilizando-se o programa Statistica/w. Para a comparação entre os grupos em relação às variáveis contínuas, adotaram-se a análise de variância (Anova) e o teste LSD para comparar os grupos dois a dois. $O$ teste $t$ de Student para amostras pareadas foi aplicado nas comparações dos resultados relativos aos segmentos mediais e referenciais dentro de cada grupo.

As variáveis correspondentes às características celulares e ao grau de lesão vascular foram analisadas por meio do teste exato de Fisher, comparando-se os grupos dois a dois. As comparações entre os resultados dos segmentos medial e referencial foram feitas por meio do teste binomial.

As variáveis contínuas foram expressas em média \pm desvio-padrão, e as variáveis categóricas, em freqüências e percentuais. As comparações foram feitas considerando-se o nível de significância corrigido de Bonferroni $(p<0,0167$ para grau de lesão e $p<0,0125$ para alterações tissulares e variáveis morfométricas).

\section{Resultados}

A média do colesterol total foi de $1.362,28 \pm 273 \mathrm{mg} / \mathrm{dl}$.

\section{Análise histológica morfométrica (mensurações quantitativas de área)}

Esta análise foi feita considerando-se as variáveis: ANI, ACM, área do vaso e razão entre ANI e ACM. Na Tabela 2,

\section{Tabela 1 Classificação anatomopatológica das alterações histológicas presentes nos diversos grupos}

\begin{tabular}{ccc}
\hline Escore & Lesão de LEI/LEE & Schwartz \\
0 & Ausente & LEI intacta, média pode estar comprimida \\
1 & Compressão & LEI lacerada, média comprimida \\
2 & Delaminação & LEI lacerada, média lacerada, LEE intacta \\
3 & Lesão focal & LEI lacerada, média lacerada com extensão para LEE \\
4 & Lesão múltipla & \\
5 & Destruição total & \\
\hline
\end{tabular}

LEI: lâmina elástica interna; LEE: lâmina elástica externa. 


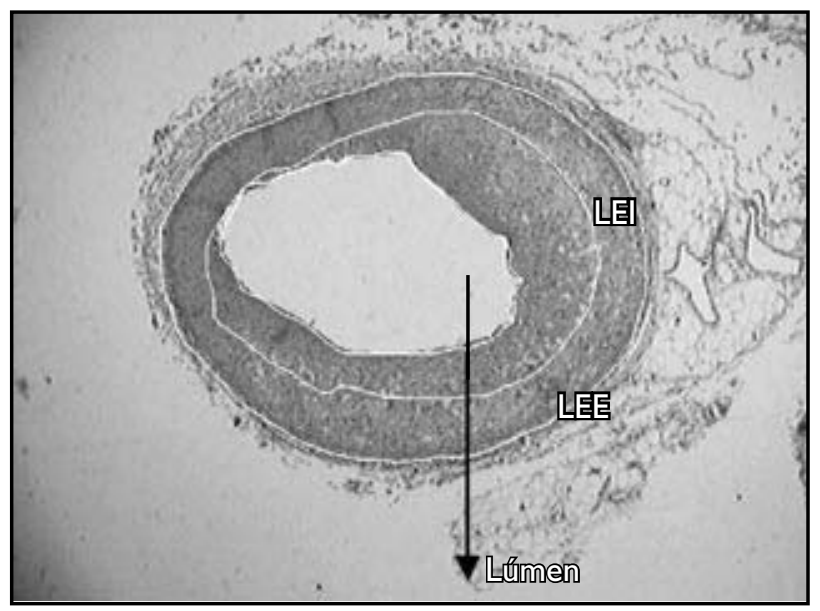

Figura 3 - Secção transversal com as medidas morfométricas delineando o lúmen e as lâminas elásticas interna e externa

são apresentados os resultados de médias e desvios-padrão destas variáveis para cada um dos grupos e valores de $p$ obtidos nas comparações de médias entre os grupos.

\section{Área neo-intimal (ANI)}

Houve significativa redução da área da neo-íntima no Gl 15Gy quando em comparação com os outros grupos. Houve diferença estatística entre os grupos com $p<0,0001$ pela Anova. Na comparação dos grupos dois a dois, constatou-se diferença estatisticamente significante entre GC e Gl 15Gy ( $p=0,0007)$, entre os GC e Gl 60Gy ( $p=0,0121)$ e entre GI 15Gy e Gl 60Gy $(p<0,0001)$ pelo teste LSD. Em média, a ANI do Gl 15Gy é 2,5 vezes menor do que a ANI do GC e 3,6 vezes menor do que a ANI do GI 60Gy. O GC é 1,4 vez menor do que o GI 60Gy (Tabela 2 e Figura 4).

\section{Área da camada média (ACM)}

O Gl 60Gy teve a maior ACM. Na comparação entre os grupos em relação à ACM foram encontradas diferenças estatisticamente significantes entre GC e Gl 60Gy

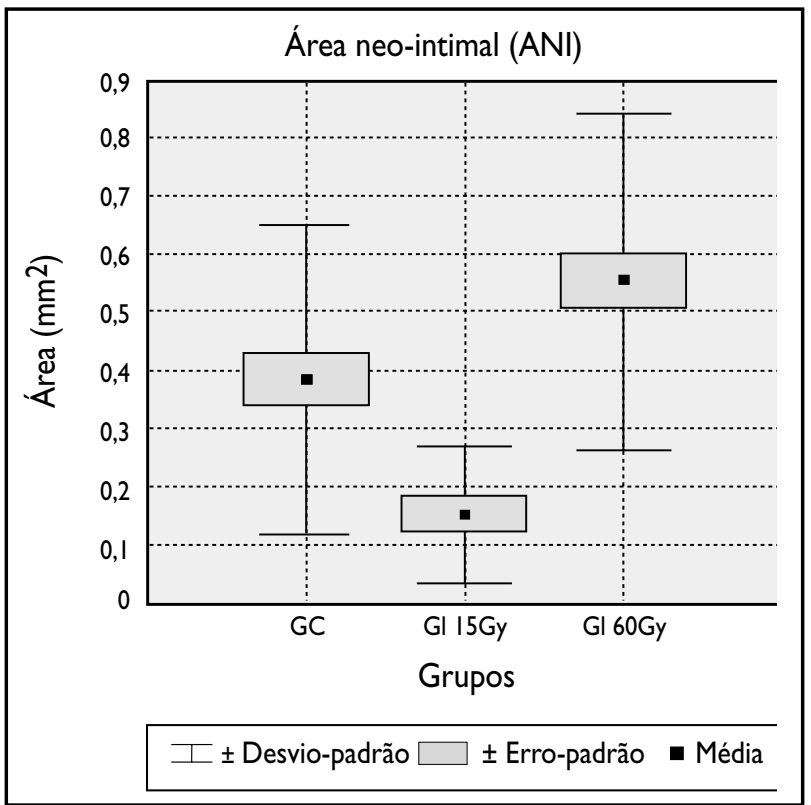

Figura 4 - Análise da área neo-intimal: comparação entre os grupos

$(p<0,0001)$ e entre GI 15Gy e Gl 60Gy $(p<0,0001)$. Não houve diferença significativa na comparação entre GC e Gl 15Gy $(p=0,0516)$.

Em média, a ACM do Gl 15Gy é 1,3 vez menor do que o GC e 1,9 vez menor do que o Gl 60Gy. No GC a ACM é 1,5 menor do que o Gl 60Gy (Figura 5).

\section{Relação entre ANI e ACM}

A razão entre $\mathrm{ANI}$ e ACM foi menor no Gl 15Gy, com diferença estatística entre os grupos $(p=0,0018)$. A razão entre ANI e ACM apresentou diferença estatisticamente significante entre GC e GI 15Gy $(p=0,0016)$, e entre Gl 15Gy e Gl 60Gy ( $p=0,001)$, o mesmo não ocorrendo quando se compararam GC e Gl 60Gy. Em média, a razão ANI/ACM é 1,8 vez menor no Gl 15Gy em relação ao GC e 1,9 vez ao Gl 60Gy. A relação média entre o GC e o Gl 60Gy foi de apenas 1,02 vez (Figura 6).

\section{Médias e desvios-padrão (em $\mathrm{mm}^{2}$ ) das variáveis morfométricas para cada grupo e valores}

Tabela 2 de $p$ nas comparações de médias entre os grupos

\begin{tabular}{lcccc}
\hline Variável & GC $(n=36)$ & GI 15Gy $(n=14)$ & GI 60Gy $(n=36)$ & Valor de $p^{*}$ \\
ANI & $0,38 \pm 0,26$ & $0,15 \pm 0,11$ & $0,55 \pm 0,28$ & $<0,0001$ \\
ACM & $0,43 \pm 0,18$ & $0,33 \pm 0,08$ & $0,64 \pm 0,19$ & $<0,0001$ \\
ANI/ACM & $0,83 \pm 0,43$ & $0,44 \pm 0,27$ & $0,85 \pm 0,35$ & 0,0018 \\
Área do vaso & $1,94 \pm 0,78$ & $1,55 \pm 0,39$ & $2,49 \pm 0,73$ & $<0,0001$ \\
\hline
\end{tabular}

GC: grupo controle; GI 15Gy: grupo irradiado 15Gy; GI 60Gy: grupo irradiado 60Gy; ANI: área neo-intimal; ACM: área da camada média; ANI/ACM: razão entre a área neointimal e a área da camada média.

*Anova. 


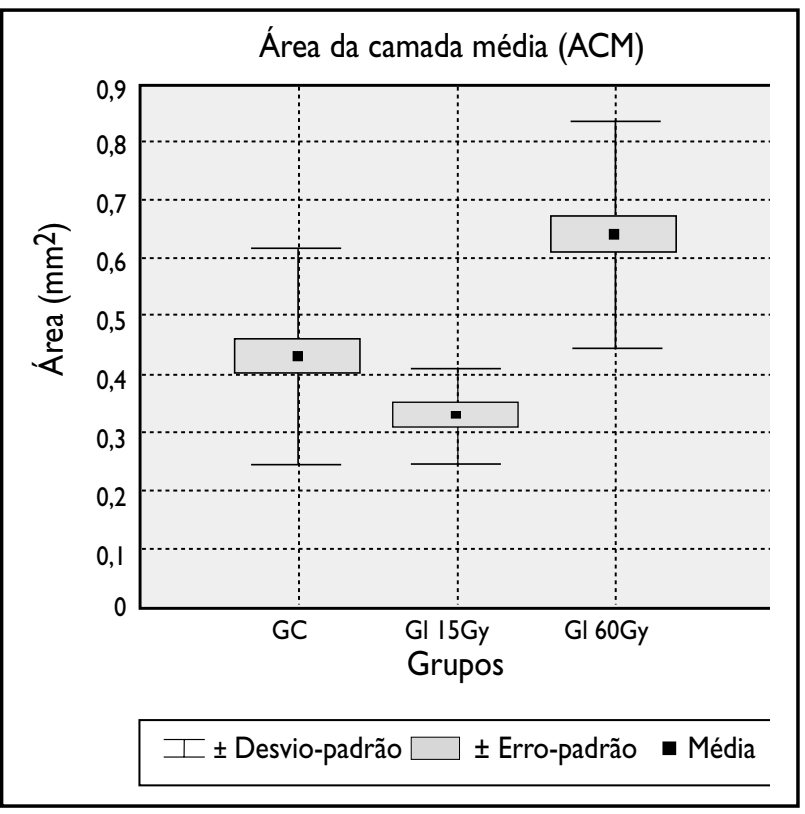

Figura 5 - Análise da área da camada média: comparação entre os grupos

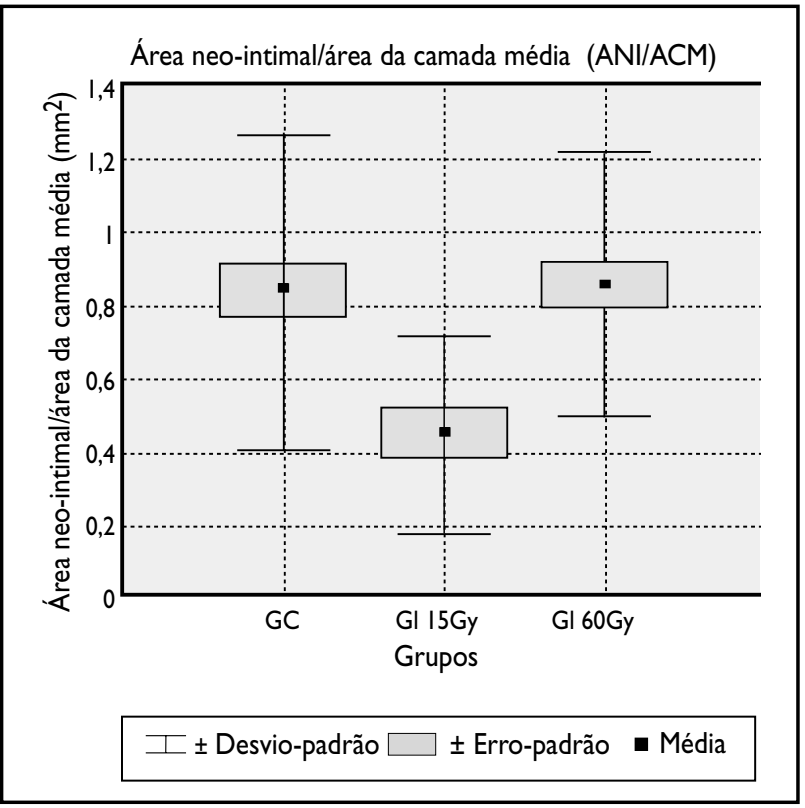

Figura 6 - Análise da razão entre a área neo-intimal e a área da camada externa

\section{Área do vaso}

A maior área do vaso foi encontrada no Gl 60Gy, com $2,49 \pm 0,73 \mathrm{~mm}^{2}$, que teve uma proporção $38 \%$ maior do que o Gl 15Gy e 22\% do que o GC. A Anova mostrou significância estatística, $\operatorname{com} p<0,0001$ (Tabela 2). Considerando-se a análise grupo a grupo, houve diferença estatística entre os grupos irradiados e entre o Gl 60Gy e o controle, com valores de $p=0,0001$ e $p=0,0014$, respectivamente, pela análise LSD.

\section{Graus de lesões vasculares}

O grau de lesão vascular analisado pela classificação de Schwartz RS et al. ${ }^{9}$ (Tabela 1) foi mais freqüente no Gl 60Gy, com diferença estatística entre o CG $(p=0,0046)$ e o Gl 15Gy $(p=0,0391)$ (Tabela 3). Entre os segmentos medial e referencial do vaso, o Gl 60Gy demonstrou diferença significativa $(p=0,0020)$ (Tabela 4).

Utilizando a classificação para as lesões da LEI (Tabela 1), obteve-se maior grau de lesão nos segmentos mediais dos três grupos, porém na análise de grupo versus grupo não houve diferença estatística (Tabelas 5 e 6).

Para análise do grau de lesão da LEE foi empregada a classificação semelhante à LEl (Tabela 1). Comparando-se grupo versus grupo, foi observado maior grau de lesão da LEE para o segmento medial no Gl 60Gy, com diferença estatística entre o GC $(p=0,0086)$ e o GI 15Gy $(<0,0001)$. Não houve diferença entre o GC e o Gl 15Gy (Tabela 7). Houve maior grau de lesão nos segmentos mediais do GC $(p=0,0078)$ e do Gl 60Gy $(p=0,0002)$ em relação ao segmento referencial (Tabela 8).

\section{Análise histológica qualitativa}

As variáveis qualitativas observadas foram células xantomatosas, tecidos hialino e amorfo, proliferação vascular e tecido de granulação e fibrose.

A proliferação vascular foi predominante no $\mathrm{Gl} 60 \mathrm{~Gy}$, presente em $30,56 \%$ dos casos. No GC ocorreu em $11,11 \%$. Houve diferença estatisticamente significativa quando comparados os Gl 15Gy e o Gl 60Gy, com $p=0,0221$. A comparação dos demais grupos não apresentou diferença estatística. Os valores estão especificados na Tabela 9.

Os tecidos de granulação e fibrose estiveram presentes em $27,78 \%$ no GC, $71,43 \%$ no Gl 15 Gy e $63,89 \%$ no Gl 60Gy. Na comparação entre os grupos observou-se significância estatística entre GC e Gl 15Gy $(p=0,0089)$ e entre GC e Gl 60Gy ( $p$ $=0,0042$ ). As freqüências e os percentuais são demonstrados na Tabela 9 e o corte histológico, na Figura 7.

A presença de células xantomatosas de grau moderado/acentuado foi observada principalmente no Gl 60Gy em $86,11 \%$ das artérias e em $33,33 \%$ no GC, tendo uma diferença estatisticamente significativa entre esses grupos, com $p<0,0001$. Quando comparados os grupos de artérias irradiadas, o Gl 60Gy apresentou predominância sobre o Gl 15Gy, com 86,11\% e 14,29\%, respectivamente, apresentando diferença estatisticamente significativa, com $p<0,0001$, pelo teste de Fisher. Não houve diferença entre 
Tabela 3 Grau de lesão do vaso conforme os segmentos do vaso ${ }^{9}$

\begin{tabular}{|c|c|c|c|c|c|c|}
\hline \multirow[b]{2}{*}{ Grau de lesão } & \multicolumn{3}{|c|}{ Medial } & \multicolumn{3}{|c|}{ Referencial } \\
\hline & $\begin{array}{c}G C \\
(n=36)\end{array}$ & $\begin{array}{l}\text { GI } 15 \mathrm{~Gy} \\
(n=14)\end{array}$ & $\begin{array}{l}\text { GI 60Gy } \\
(n=36)\end{array}$ & $\begin{array}{c}G C \\
(n=36)\end{array}$ & $\begin{array}{l}\text { GI 15Gy } \\
(n=14)\end{array}$ & $\begin{array}{l}\text { GI 60Gy } \\
(n=36)\end{array}$ \\
\hline$<2$ & $33(91,67 \%)$ & $13(92,86 \%)$ & $22(61,11 \%)$ & $35(97,22 \%)$ & $14(100 \%)$ & $32(88,89 \%)$ \\
\hline \multirow[t]{6}{*}{$\geq 2$} & $3(8,33 \%)$ & $1(7,14 \%)$ & $14(38,89 \%)$ & $1(2,78 \%)$ & $0(0 \%)$ & $4(11,11 \%)$ \\
\hline & \multicolumn{3}{|c|}{ Grupos comparados } & \multicolumn{3}{|l|}{ Valor de $p^{*}$} \\
\hline & \multirow{2}{*}{\multicolumn{2}{|c|}{ GC $x$ GI 15Gy }} & Medial & \multicolumn{2}{|c|}{ Referencial } & \\
\hline & & & 1 & \multicolumn{2}{|c|}{1} & \\
\hline & \multicolumn{2}{|c|}{ GC $\times$ GI 60Gy } & 0,0046 & \multirow{2}{*}{\multicolumn{2}{|c|}{0,357}} & \\
\hline & \multicolumn{2}{|c|}{ GI 15Gy $x$ GI 60Gy } & 0,0391 & & & \\
\hline
\end{tabular}

GC: grupo controle; GI 15Gy: grupo irradiado 15Gy; GI 60Gy: grupo irradiado 60Gy.

*Teste exato de Fisher.

Número de casos de acordo com o grau de lesão do vaso, dos segmentos mediais e referenciais

\section{Tabela 4 de cada grupo}

\begin{tabular}{|c|c|c|c|c|c|c|c|}
\hline & & \multicolumn{6}{|c|}{ Grau de lesão no referencial } \\
\hline & & \multicolumn{2}{|c|}{ GC $(n=36)$} & \multicolumn{2}{|c|}{ GI 15Gy $(n=14)$} & \multicolumn{2}{|c|}{ GI 60Gy $(n=36)$} \\
\hline & & $<2$ & $\geq 2$ & $<2$ & $\geq 2$ & $<2$ & $\geq 2$ \\
\hline \multirow[t]{2}{*}{ Grau de lesão no medial } & $<2$ & 33 & 0 & 13 & 0 & 22 & 0 \\
\hline & $\geq 2$ & 2 & 1 & 1 & 0 & 10 & 4 \\
\hline Valor de $p^{*}$ & & \multicolumn{2}{|c|}{0,5} & \multicolumn{2}{|c|}{1} & \multicolumn{2}{|c|}{0,002} \\
\hline
\end{tabular}

GC: grupo controle; GI 15Gy: grupo irradiado 15Gy; GI 60Gy: grupo irradiado 60Gy.

*Teste binomial.

\section{Grau de lesão da lâmina elástica interna (LEI) dos segmentos mediais e referenciais}

\begin{tabular}{|c|c|c|c|c|c|c|}
\hline \multirow[b]{2}{*}{ Grau de lesão } & \multicolumn{3}{|c|}{ Medial } & \multicolumn{3}{|c|}{ Referencial } \\
\hline & $\begin{array}{c}G C \\
(n=36)\end{array}$ & $\begin{array}{l}\text { GI 15Gy } \\
(n=14)\end{array}$ & $\begin{array}{l}\text { GI 60Gy } \\
(n=36)\end{array}$ & $\begin{array}{c}G C \\
(n=36)\end{array}$ & $\begin{array}{l}\text { GI 15Gy } \\
(n=14)\end{array}$ & $\begin{array}{l}\text { GI 60Gy } \\
(n=36)\end{array}$ \\
\hline$<2$ & $11(30,56 \%)$ & $2(14,29 \%)$ & $8(22,22 \%)$ & $21(58,33 \%)$ & $10(71,43 \%)$ & $16(44,44 \%)$ \\
\hline \multirow[t]{6}{*}{$\geq 2$} & $25(69,44 \%)$ & $12(85,71 \%)$ & $28(77,78 \%)$ & $15(41,67 \%)$ & $4(28,57 \%)$ & $20(55,56 \%)$ \\
\hline & \multirow{2}{*}{\multicolumn{2}{|c|}{ Grupos comparados }} & \multicolumn{2}{|c|}{ Valor de $p^{*}$} & & \\
\hline & & & Medial & Referencial & & \\
\hline & \multicolumn{2}{|c|}{ GC $x$ GI 15Gy } & 0,303 & 0,5218 & & \\
\hline & \multicolumn{2}{|c|}{ GC $x$ GI 60Gy } & 0,5936 & 0,3457 & & \\
\hline & \multicolumn{2}{|c|}{ GI 15Gy $x$ GI 60Gy } & 0,7042 & 0,1192 & & \\
\hline
\end{tabular}

GC: grupo controle; GI 15Gy: grupo irradiado 15Gy; GI 60Gy: grupo irradiado 60Gy.

*Teste exato de Fisher. 


\section{Número de casos de acordo com o grau de lesão da lâmina elástica interna dos segmentos}

Tabela 6

\section{medial e referencial de cada grupo}

\begin{tabular}{lccccccc}
\hline & \multicolumn{7}{c}{ Grau de lesão no referencial } \\
& \multicolumn{1}{c}{ GC $(n=36)$} & GI 15Gy $(n=14)$ & \multicolumn{2}{c}{ GI 60Gy $(n=36)$} \\
& $<2$ & $\geq 2$ & $<2$ & $\geq 2$ & $<2$ & $\geq 2$ \\
Grau de lesão no medial & $<2$ & 8 & 3 & 2 & 0 & 6 & 2 \\
Valor de $p^{*}$ & $\geq 2$ & 13 & 12 & 8 & 4 & 10 & 18 \\
\hline
\end{tabular}

GC: grupo controle; Gl 15Gy: grupo irradiado 15Gy; Gl 60Gy: grupo irradiado 60Gy.

*Teste binomial.

Tabela 7 Grau de lesão da lâmina elástica externa (LEE) dos segmentos medial e referencial de cada grupo

\begin{tabular}{|c|c|c|c|c|c|c|}
\hline \multirow[b]{2}{*}{ Grau de lesão } & \multicolumn{3}{|c|}{ Medial } & \multicolumn{3}{|c|}{ Referencial } \\
\hline & $\begin{array}{c}\mathrm{GC} \\
(n=36)\end{array}$ & $\begin{array}{l}\text { GI 15Gy } \\
(n=14)\end{array}$ & $\begin{array}{l}\text { GI 60Gy } \\
(n=36)\end{array}$ & $\begin{array}{c}G C \\
(n=36)\end{array}$ & $\begin{array}{l}\text { GI 15Gy } \\
(n=14)\end{array}$ & $\begin{array}{l}\text { GI 60Gy } \\
(n=36)\end{array}$ \\
\hline \multirow{7}{*}{$\begin{array}{l}<2 \\
\geq 2\end{array}$} & $16(44,44 \%)$ & $11(78,57 \%)$ & $5(13,89 \%)$ & $24(66,67 \%)$ & $13(92,86 \%)$ & $17(47,22 \%)$ \\
\hline & $20(55,56 \%)$ & $3(21,43 \%)$ & $31(86,11 \%)$ & $12(33,33 \%)$ & $1(7,14 \%)$ & $19(52,78 \%)$ \\
\hline & \multicolumn{3}{|c|}{ Grupos comparados } & \multicolumn{3}{|c|}{ Valor de $p^{*}$} \\
\hline & \multirow{4}{*}{\multicolumn{2}{|c|}{$\begin{array}{l}\text { GC } \times \text { GI 15Gy } \\
\text { GC } \times \text { GI } 60 \mathrm{~Gy} \\
\text { GI 15Gy } x \text { GI 60Gy }\end{array}$}} & Medial & \multirow{4}{*}{\multicolumn{2}{|c|}{$\begin{array}{c}\text { Referencial } \\
0,0783 \\
0,1582 \\
0,0035\end{array}$}} & \\
\hline & & & 0,056 & & & \\
\hline & & & 0,0086 & & & \\
\hline & & & $<0,0001$ & & & \\
\hline
\end{tabular}

GC: grupo controle; GI 15Gy: grupo irradiado 15Gy; GI 60Gy: grupo irradiado 60Gy.

*Teste exato de Fisher.

\section{Número de casos de acordo com o grau de lesão da lâmina elástica externa dos segmentos}

Tabela 8 medial e referencial de cada grupo

\begin{tabular}{|c|c|c|c|c|c|c|c|}
\hline & & \multicolumn{6}{|c|}{ Grau de lesão no referencial } \\
\hline & & \multicolumn{2}{|c|}{$\mathrm{GC}(n=36)$} & \multicolumn{2}{|c|}{$\begin{array}{l}\text { GI 15Gy }(n=14) \\
<2 \quad \geq 2\end{array}$} & \multicolumn{2}{|c|}{$\begin{array}{c}\text { GI } 60 \mathrm{~Gy}(n=36) \\
<2\end{array}$} \\
\hline Grau de lesão no medial & $\begin{array}{l}<2 \\
\geq 2\end{array}$ & $\begin{array}{c}16 \\
8\end{array}$ & $\begin{array}{c}0 \\
12\end{array}$ & $\begin{array}{c}11 \\
2\end{array}$ & $\begin{array}{l}0 \\
1\end{array}$ & $\begin{array}{c}4 \\
13\end{array}$ & $\begin{array}{c}0 \\
19\end{array}$ \\
\hline Valor de $p^{*}$ & & \multicolumn{2}{|c|}{0,0078} & \multicolumn{2}{|c|}{0,5} & \multicolumn{2}{|c|}{0,0002} \\
\hline
\end{tabular}

GC: grupo controle; Gl 15Gy: grupo irradiado 15Gy; Gl 60Gy: grupo irradiado 60Gy.

*Teste binomial.

Alterações tissulares encontradas nos diversos grupos estudados: frequîencias e intervalos de

Tabela 9 95\% de confiança para as proporções de presença

\begin{tabular}{lcccc}
\hline Variável & GC $(n=36)$ & GI 15Gy $(n=14)$ & GI 60Gy $(n=36)$ & Valores de $p^{*}$ \\
Proliferação vascular & $4(0,01 ; 0,21)$ & 0 & $11(0,16 ; 0,46)$ & $0,0221^{*}$ \\
Tecido de granulação e fibrose & $10(0,13 ; 0,42)$ & $10(0,48 ; 0,95)$ & $23(0,48 ; 0,8)$ & $0,0089^{* *} 0,002^{* * *}$ \\
Célula xantomatosa & $12(0,18 ; 0,49)$ & $2(0 ; 0,33)$ & $31(0,75 ; 0,97)$ & $<0,0001^{*} 0,0001^{* * *}$ \\
Material amorfo e hialino & $5(0,03 ; 0,25)$ & 0 & $21(0,42 ; 0,74)$ & $0,0001^{*} 0,0002^{* * *}$ \\
\hline
\end{tabular}

GC: grupo controle; GI 15Gy: grupo irradiado 15Gy; GI 60Gy: grupo irradiado 60Gy. Valor de p: teste exato de Fisher.

${ }^{*} \mathrm{Cl} 15 \mathrm{~Gy} \times \mathrm{Gl}$ 60Gy; ${ }^{* *} \mathrm{CC}$ x Gl 15Gy; ${ }^{* *} \mathrm{CC} \times$ Gl 60Gy. 
o GC e o Gl 15Gy. Os valores estão especificados na Tabela 8 e os cortes histológicos, nas Figuras 8 e 9 .

O material amorfo e hialino foi observado no GC em $13,89 \%$ dos casos e em $58,33 \%$ no Gl 60 Gy. Não ocorreu alteração no Gl 15Gy. Houve significância estatística quando comparados os GC e Gl 60Gy $(p=0,0002)$ e os grupos Gl 15Gy e Gl 60Gy ( $p=0,0001)$. Entre os grupos GC e GI15Gy não houve significância. As freqüências e percentuais são demonstrados na Tabela 9.

\section{Discussão}

Neste estudo, utilizou-se uma classificação de lesão vascular modificada de Schwartz RS et al. ${ }^{(9)}$ e observaram-se graus moderados e acentuados de lesão, principalmente nos segmentos mediais das artérias ilíacas na dose de 60Gy (local da insuflação do balão) em relação aos segmentos referenciais, com significância estatística quando em comparação com os outros dois grupos. Esses graus de lesão denotam a LEl e a LEE laceradas, comunicando a camada média com a neoíntima e a adventícia. Essas alterações sugerem que o efeito não deve ser somente pelo trauma do balão, uma vez que os outros grupos (GC e Gl 15Cy) também foram submetidos à mesma pressão, mas sim pela elevada dose de irradiação, que favoreceu as modificações estruturais da LEl e da LEE.

A LEI tem a função de manter a estrutura do vaso, servindo como barreira entre a íntima e a camada média e impedindo a migração de células e macromoléculas. A sua estrutura é composta de fibras elásticas delgadas com fenestrações que variam conforme as espécies ${ }^{(10)}$. A presença de células xantomatosas freqüentes no espaço intimal é citada como característica típica de radiolesão quando se associa à hipercolesterolemia. Kwon et al.(11), estudando as mudanças estruturais da LEI em suínos hipercolesterolêmicos, notaram que há aumento da comunicação entre a íntima e a camada média. Semelhantes achados foram observados neste estudo, com as alterações da LEI associadas à significativa comunicação entre a íntima e

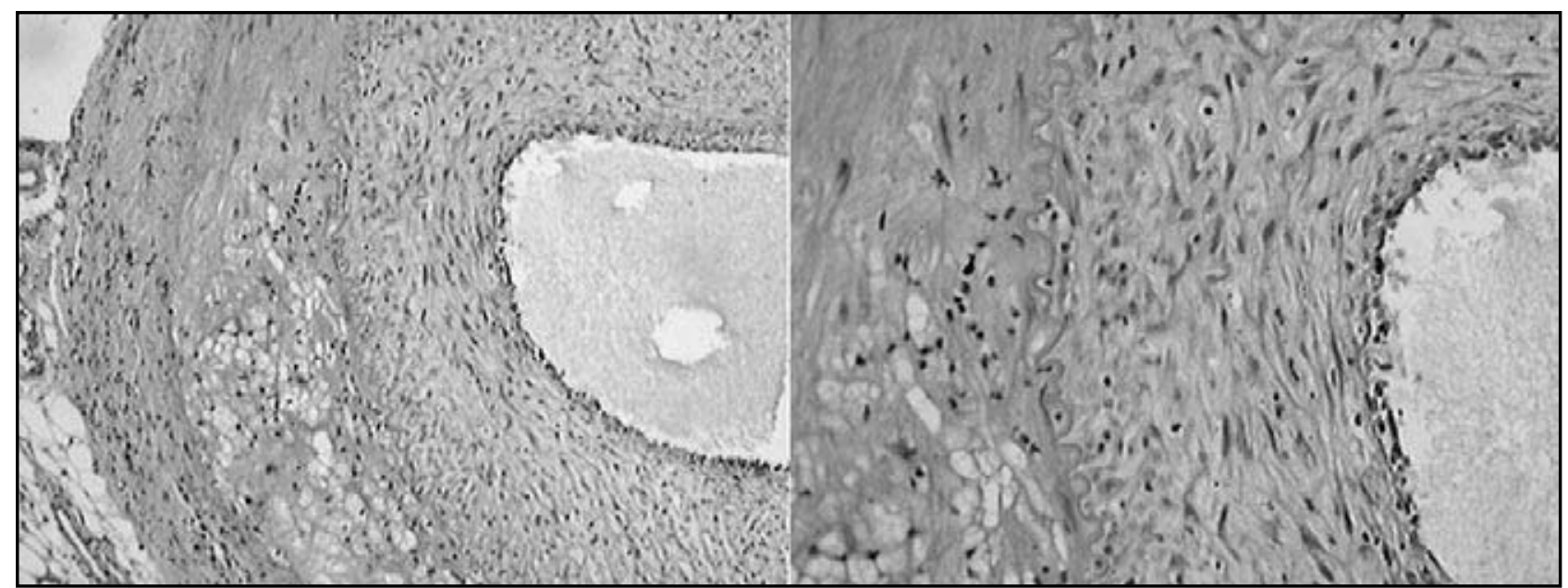

Figura 7 - Artéria irradiada 60Gy, com tecido de granulação e fibrose

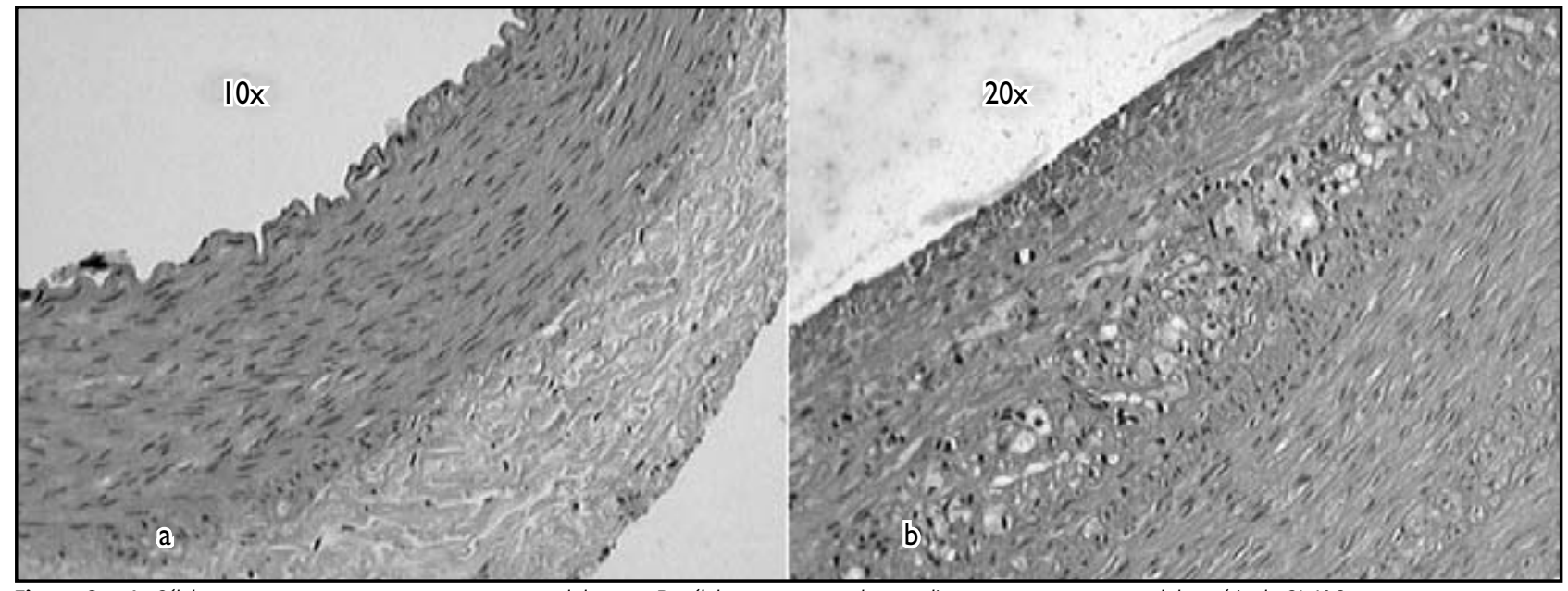

Figura 8 - A: Célula xantomatosa ausente em corte transversal de aorta; B: célula xantomatosa de grau discreto em corte transversal de artéria do GI 60Gy 


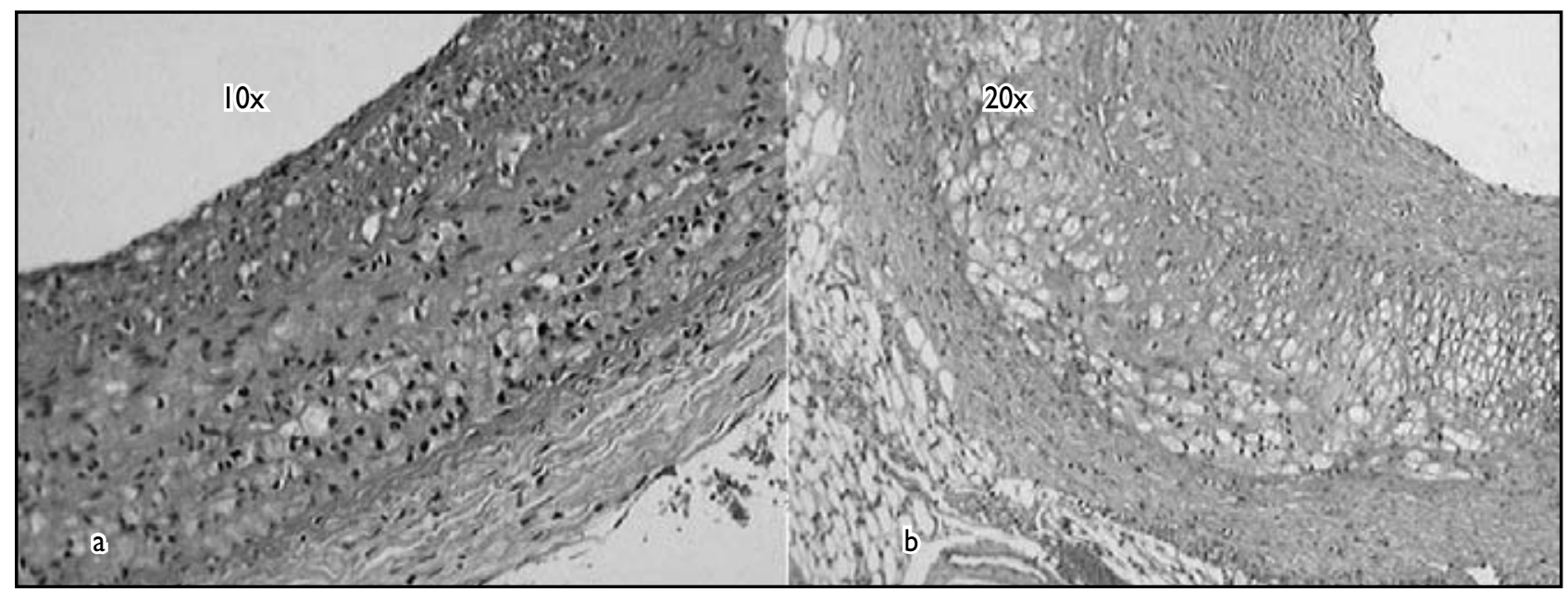

Figura 9 - A: Célula xantomatosa de grau moderado em corte transversal de artéria do GC; B: célula xantomatosa de grau acentuado em corte transversal de artéria do GI 60Gy

a camada média sendo evidenciadas pelo aumento da presença de células xantomatosas na camada média, local que não é próprio destas células. As artérias irradiadas com 60Gy apresentaram o predomínio destas células no grau moderado e acentuado, sendo observado em $86,11 \%$ dos segmentos arteriais analisados e somente em $33,33 \%$ no grupo controle e $14,29 \%$ no grupo irradiado com 15Gy, com significância estatística entre os grupos $(p<0,0001)$. O aumento da área da camada média reflete tais alterações estruturais. Na análise do grau de comprometimento lesional da LEl, verificou-se que no grupo 60Gy foi em média 13\% maior que nos outros grupos.

Estudos têm demonstrado a utilidade da irradiação com emissores beta na prevenção da reestenose ${ }^{(12)}$. Waksman et al. (13) utilizaram doses de 7, 14, 28 e 56Gy de 90Sr/Y. As doses foram calculadas para atingir $2 \mathrm{~mm}$ da parede do vaso e resultaram em adequada inibição da proliferação intimal sem necrose das camadas da artéria. Neste estudo, o Gl 60Gy teve $63,89 \%$ de fibrose, em comparação com $27,78 \%$ no GC e $71,435 \%$ no Gl 15Gy. Esses achados sugerem a presença de lesão ocasionada pela irradiação, com a provável penetração exacerbada com a dose elevada do ${ }^{153} \mathrm{Sm}$ de $60 \mathrm{~Gy}$. Tal observação é ressaltada ao se analisar o grau de lesão da LEE, que foi $48,5 \%$ maior no Gl $60 \mathrm{~Gy}$ do que no Gl 15Gy e $31 \%$ maior do que no GC. Este fato reforça a hipótese de radiolesão, e não lesão pelo trauma do balão de angioplastia. Tal achado corrobora a literatura, sugerindo que esta fibrose seja atribuída à radiação, peculiar aos radioisótopos. Entretanto, com a dose de 15Gy, não foram observadas anormalidades celulares e histológicas encontradas com a dose de 60Gy, o que pode sugerir que estes fenômenos são dose-resposta. Estes achados são discutidos por Denham e Hauer-Jensen ${ }^{(14)}$. Eles descrevem a diferença da lesão arterial pelo trauma e pela radiação, sendo que esta última envolve uma produção excessiva de citocinas, o acúmulo exagerado de matriz extracelular e a conseqüente fibrose. Trott e Kamprad(15) estudaram os efeitos antiinflamatórios e pró-inflamatórios da radiação e assinalam a forte correlação com a dose aplicada. Isto pode explicar a diferença encontrada entre os dois grupos de artérias irradiadas neste experimento, sendo que com a dose de 15Gy observou-se fibrose semelhante à ocorrida com a dose de 60Gy, porém sem a presença de células xantomatosas, material amorfo hialino e alterações nas áreas intimal e da camada média observadas na dose de 60Gy.

A principal característica do dano vascular após balão de angioplastia é a hiperplasia neo-intimal, que é um tipo de lesão fibrocelular, conferindo limitação ao fluxo sangüíneo. As características principais são a migração das células da camada média, a alteração fenotípica e a secreção de matriz celular ${ }^{(16)}$. Estas características representam uma das principais causas da reestenose em humanos submetidos à angioplastia por balão, além do recolhimento elástico da parede arterial. A braquiterapia intravascular nos últimos anos tem sido utilizada como uma das principais terapêuticas da reestenose através de doses específicas e atuando como um supressor do crescimento celular ${ }^{(17,18)}$. Semelhantes observações foram evidenciadas neste estudo, no qual encontraram-se resultados significativos na inibição neo-intimal com a utilização da dose de 15Gy. Pela correlação de Pearson, observamos uma relação direta e estatisticamente significativa entre a ANI e a ACM nos três grupos, isto é, como no Gl 15Gy houve menor proliferação intimal, ocorreu menor dimensão da camada média. Encontramos também, nesse grupo, significativa diminuição da área do vaso. Esse resultado foi um dos mais relevantes deste experimento, o que evidencia o benefício da dose de 15Gy em inibir a reprodução celular. Estes achados corroboram outros estudos, utilizando a irradiação vascular 
com outros radioisótopos, tanto com a técnica de emissão gama, como com o uso de partículas beta ${ }^{(19-21)}$. A utilização em humanos mostrou a mesma eficácia dos modelos experimentais, usados inicialmente com irradiação gama e posteriormente com o uso de partículas beta ${ }^{(16)}$.

\section{Conclusões}

A irradiação intra-arterial com a dose absorvida de ${ }^{(153)}$ Sm de 15Gy foi adequada para reduzir a área neo-intimal, a área da camada média e a área do vaso. Na dose elevada de 60Gy foi observado aumento significativo destes dados morfométricos, além da presença significativa de padrões celulares anormais, tais como células xantomatosas e material amorfo hialino, dados estes compatíveis com efeitos de radiolesão.

Não foram utilizadas doses intermediárias do ${ }^{153} \mathrm{Sm}$, que provavelmente teriam melhor efeito do que a dose de 15Gy e sem os efeitos deletérios observados na dose elevada de 60Gy utilizada neste estudo.

\section{Referências}

I. SHEPPARD, R.; EISENBERG, M. J. Intracoronary radiotherapy for restenosis. N Engl J Med, v. 344, p. 295-7, 2001.

2. SERRUYS, P.W. et al. A comparison of ballon-expandable-stent implantation with ballon angioplasty in patients with coronary artery disease. $N$ Engl J Med, v. 33I, p. 489-95, 1994.

3. CLOWES, A. W.; SCHWARTZ, S. M. Significance of quiescent smooth muscle migration in the injured rat carotid artery. Circ Res, v. 56, p. 139-45, 1985.

4. GALIS, Z. S.; KHATRI, J. J. Matrix metalloproteinases in vascular remodeling and atherogenesis: the good, the bad, and the ugly. Circ Res, v. 90, p. 25I-62, 2002.

5. SCHWARTZ, S. M.; deBLOIS, D.; O'BRIEN, E. R. M. The Intima: soil for atherosclerosis and restenosis. Circ Res, v. 77 , p. 445-65, 1995.

6. TEIRSTEIN, P. S. et al. Catheter-based radiotherapy to inhibit restenosis after coronary stenting. N Engl J Med, v. 336, p. 1697-703, 1997.

7. SALAME, M.Y. et al. Intracoronary radiation therapy. Eur Heart J, v. 22, p. 629-47, 2001.

8. SAPIRSTEIN, W.; ZUCKERMAN, B.; DILLARD, J. FDA approval of coronary-artery brachytherapy. N Engl J Med, v. 344, p. 297-9, 2001.

9. SCHWARTZ, R. S. et al. Artery size, neointima, and remodeling: time for some standards. J Am Coll Cardiol, v. 32, p. 2087-94, 1998.

10.WONG, L. C.Y.; LANGILLE, B. L. Development remodeling of the internal elastic lamina of rabbit arteries. Circ Res, v. 78, p. 799-805, 1996.

I I. KWON, H. M. et al. Experimental hypercholesterolemia induces ultrastructural changes in the internal elastic lamina of porcine coronary arteries. Atherosclerosis, v. 139, p. 283-9, 1998.

12. WAKSMAN, R. et al. Intracoronary radiation before stent implantation inhibits neointima formation in stented porcine coronary arteries. Circulation, v. 92, p. 1383-6, 1995.

13. WAKSMAN, R. et al. Intracoronary low-dose â-irradiation inhibits neointima formation after coronary artery balloon injury in the swine restenosis model. Circulation, v. 92 p. 303।-52, 1995.

14. DENHAM, J. W.; HAEUR-JENSEN, M. The radiotherapeutic injury-a complex "wound". Radiother Oncol, v. 63, p. 129-45, 2002.

15. TROTT, K. R.; KAMPRAD, F. Radiobiological mechanisms of anti-inflammatory radiotherapy. Radiother Oncol, v. $5 \mathrm{I}$, p. 197-203, 1999.

16. MEERKIN, D. et al. Effects of intracoronary beta-radiation therapy after coronary angioplasty: an intravascular ultrasound study. Circulation, v. 99, n. 13, p. 1660-5, 6 abr. 1999.

17. GRISE, M.A. et al. Five-year clinical follow-up after intracoronary radiation: results of a randomized clinical trial. Circulation, v. 105, p. 2737-40, 2002.

18. JOHNSON, G. J.; GRIGGS, T. R.; BADIMON, L. The utility of animal models in the preclinical study of interventions to prevent human coronary artery restenosis: analysis and recommendations. On behalf of the Subcommittee on Animal, Cellular and Molecular Models of Thrombosis and Haemostasis of the Scientific and Standardization Committee of the International Society on Thrombosis and Haemostasis. Thromb Haemost, v. 81, p. 835-43, 1999.

19. JONES, B. Intracoronary artery radiation. Br J Radiol, v. 72, p. 1033-6, 1999.

20. KNAPP Jr., F. F.; SPENCER, R. H.; KROPP, J. Intravascular radiation therapy with radioactive liquid-filled ballons for inhibition of restenosis after angioplasty: a new opportunity for nuclear medicine? J Nucl Med, v. 42, p. I384-7, 2001.

21. HEHRLEIN, C. et al. Pure $\beta$-particle emitting stents inhibit neointima formation in rabbits. Circulation, v. 93, p. 64I-5, 1996.

Endereço para correspondência

Lúcia de Noronha

Laboratório de Patologia Experimental

Rua Imaculada Conceição 1.155 - Prado Velho

CEP 80215-901 - Curitiba-PR

Caixa Postal 16210 